\title{
Time-Dependent Multiphoton Ionization of Xenon in the Soft-X-Ray Regime
}

\author{
N. Gerken, ${ }^{1}$ S. Klumpp, ${ }^{1}$ A. A. Sorokin,${ }^{2,3}$ K. Tiedtke, ${ }^{2}$ M. Richter, ${ }^{4}$ V. Bürk, ${ }^{1}$ K. Mertens, ${ }^{1}$ P. Juranić,,${ }^{2}$ and M. Martins ${ }^{1}$ \\ ${ }^{1}$ Universität Hamburg, Institut für Experimentalphysik, Luruper Chaussee 149, 22761 Hamburg, Germany \\ ${ }^{2}$ Deutsches Elektronen-Synchrotron, DESY, Notkestraße 85, 22603 Hamburg, Germany \\ ${ }^{3}$ Ioffe Physico-Technical Institute, Polytekhnicheskaya 26, 194021 St. Petersburg, Russia \\ ${ }^{4}$ Physikalisch-Technische Bundesanstalt, PTB, Abbestraße 2-12, 10587 Berlin, Germany
}

(Received 29 October 2013; published 30 May 2014)

\begin{abstract}
The time-dependent multiphoton ionization of xenon atoms is studied with femtosecond pulses in the excitation range of the $4 d$ giant resonance at the photon energy of $93 \mathrm{eV}$. Benefiting from a new operation mode of the free electron laser FLASH, the measurements are performed with varying pulse durations. A strong dependence of the ion charge distribution on the pulse duration allows the different multiphoton mechanisms behind the multiple photoionization of xenon to be disentangled up to a charge state of $\mathrm{Xe}^{10+}$. The results up to $\mathrm{Xe}^{8+}$ are well explained by sequences of single photon, multiphoton, and Auger processes, but higher charge state generation suggests the need for collective electron multiphoton excitations.
\end{abstract}

The highly intense and ultrashort radiation pulses of the new free electron laser (FEL) facilities in Europe [1,2], the United States [3], and Japan [4,5] can significantly extend the study of interaction of $\mathrm{x}$ rays with matter to the nonlinear regime of multiphoton processes. A number of fascinating FEL experiments of multiphoton ionization have already been carried out on solids, clusters, molecules, and on free atoms [[6,7,8,9], and references therein]. Whereas in the regime of optical femtosecond lasers, simultaneous multiphoton, above-threshold, and strongfield ionization are the mechanisms of relevance [10,11], the nonlinear interaction of soft and hard $\mathrm{x}$ rays with atoms at photon energies above the first ionization threshold has been shown to be dominated by sequential processes in which an excited atom or ion created in a preceding step represents the target for a subsequent step [12-20]. For higher ionization charges and ionization thresholds which exceed the photon energy, the mechanisms may be rather complex because simultaneous multiphoton ionization processes come into play for the higher steps of an ionization sequence [12,21].

A prime example is the multiphoton multiple ionization of $\mathrm{Xe}$ in the vicinity of the so-called $4 d$ giant resonance in the extreme ultraviolet (EUV) between $90 \mathrm{eV}$ and $110 \mathrm{eV}$ photon energy where charge states up to $\mathrm{Xe}^{21+}$ were observed at irradiance levels in the order of $10^{16} \mathrm{~W} / \mathrm{cm}^{2}$ [22]. A sequential scheme requires 19 steps and almost 60 EUV photons to generate $\mathrm{Xe}^{21+}$ from atomic $\mathrm{Xe}$, and more than a single photon is required for each step from $\mathrm{Xe}^{7+}$ on. The role of the $4 d$ giant resonance and the impact of collective electron excitation on this particular behavior are still-open scientific questions of fundamental importance [22-26]. Since the different multiphoton schemes depend in different ways on the FEL pulse duration, as has recently been summarized [27], photoionization experiments at varying pulse duration may help to disentangle the underlying mechanisms. Experiments at the Linac Coherent Light Source (LCLS) have demonstrated that sequences of inner-shell excitation in the harder x-ray regime and successive refilling via Auger decay is the dominating mechanism for the complete stripping of the electrons on $\mathrm{Ne}$ atoms [16]. In the present work, we have investigated the much more complex multiphoton multiple ionization of $\mathrm{Xe}$ at lower photon energy in the EUV by time-dependent measurements at the free electron laser FLASH, benefiting from a new operation mode with varying pulse duration within a FEL bunch train in the range from $150 \mathrm{fs}$ to $250 \mathrm{fs}$. The data were compared to the already-reported results obtained at much shorter pulse duration in the order of $10 \mathrm{fs}$ to 20 fs [22]. This first time-dependent multiphoton ionization study in the EUV demonstrates that the generation of the Xe charge states is explained by a complex scheme of different multiphoton mechanisms which can be distinguished.

The experiments were performed at a target pressure of around $10^{-7}$ mbar using the BL2 beam line of FLASH with a FWHM focal diameter of $(20 \pm 5) \mu \mathrm{m}[28,29]$. The photon energy of $93 \mathrm{eV}$ was chosen to compare current data with the former results of the Xe multiphoton ionization obtained at $(2.6 \pm 0.5) \mu \mathrm{m}$ focal diameter [22]. The FEL beam was arranged in $300 \mu$ s long bunch trains at a $10 \mathrm{~Hz}$ repetition rate, with each train consisting of 30 individual FEL pulses. The individual pulses were separated by $10 \mu \mathrm{s}$ and had statistically fluctuating pulse intensities and shapes. The pulse duration was estimated to be at least $150 \mathrm{fs}$ and at most $250 \mathrm{fs}$ with a mean pulse duration of about 200 fs. This estimation is based on the following three different independent measurement methods: (1) an 
autocorrelation method using two movable mirrors [30,31], (2) measurement of the spectral distribution of the FEL radiation and estimation of the pulse duration from the number of modes [32], and (3) estimation of the pulse duration of the FEL radiation from the length of the electron bunches [33]. The pulse energy of the FEL was monitored by a gas monitor detector [34]. Ion time-of-flight (iToF) spectra were taken individually using a transient recorder to be able to sort the data with respect to pulse position within the bunch train and to pulse energy.

Figure 1 shows the iToF spectra of Xe atoms obtained at different pulse duration $\tau$, pulse energy $W$, and beam cross section $A$. The spectrum in Fig. 1(a) is measured at short pulse duration of $(15 \pm 5)$ fs taken from [22], whereas the spectrum sketched in (b) was obtained at much longer pulses in the order of $200 \mathrm{fs}$. For a distinct comparison, the plots were taken at selected pulse energies so that the radiant exposure $H=W / A$ had almost the same value of $1.45 \mathrm{~J} / \mathrm{cm}^{2}$ for both of them. Dividing the radiant exposure of a radiation pulse by the pulse duration yields the peak irradiance $E=H / \tau=W /(A \tau)$, which represents the key quantity of the radiation field to describe the rate of a simultaneous $n$-photon process according to the general power law of low-order perturbation theory $[27,35]$,

$$
\dot{N}^{(n)}=N_{0} \sigma^{(n)}\left(\frac{E}{\hbar \omega}\right)^{n}=N_{0} \sigma^{(n)}\left(\frac{H}{\tau \hbar \omega}\right)^{n},
$$

with the number of targets within the interaction volume $N_{0}$, the respective generalized $n$-photon ionization cross section $\sigma^{(n)}$, and the photon energy $\hbar \omega$. At constant exposure $H$, simultaneous multiphoton processes rise with

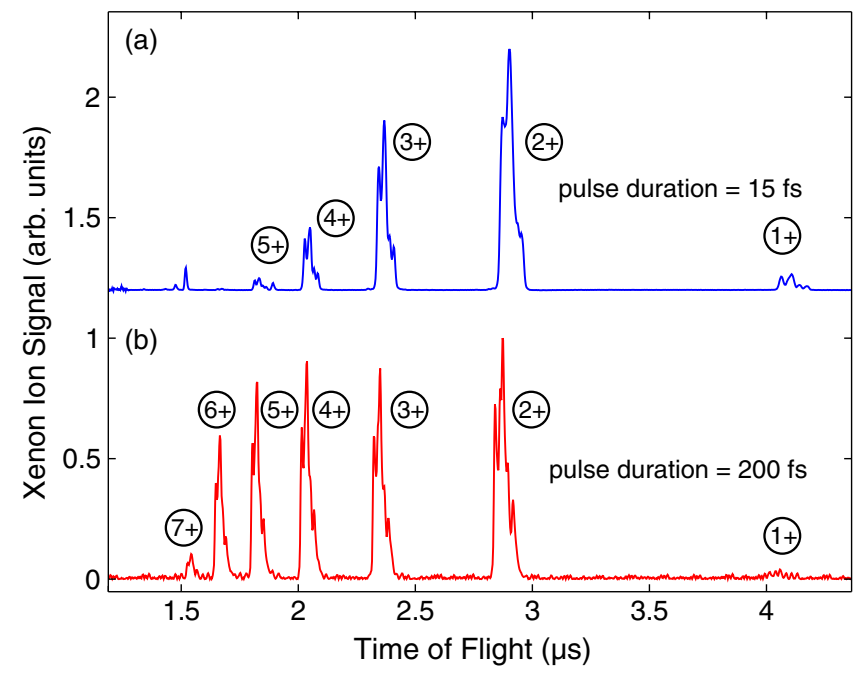

FIG. 1 (color online). Ion time-of-flight (iToF) spectra of ionized $\mathrm{Xe}$ atoms at high pulse intensity for different pulse durations at the same radiant exposure of $1.45 \mathrm{~J} / \mathrm{cm}^{2}$ : (a) for short pulses of $(15 \pm 5)$ fs measured at the irradiance of $1.4 \times$ $10^{14} \mathrm{~W} / \mathrm{cm}^{2}$ [22] and (b) for long pulses in the order of $200 \mathrm{fs}$ measured at $7.4 \times 10^{12} \mathrm{~W} / \mathrm{cm}^{2}$. shorter pulse duration $\tau$, which generally leads to higher charge states. However, in Fig. 1 the opposite behavior is demonstrated. At constant exposure, the mean charge is lower at shorter pulses, indicating that straight simultaneous multiphoton ionization may not be the underlying mechanism. This is not surprising because the multiphoton ionization at $93 \mathrm{eV}$ photon energy and radiant exposure levels in the order of a few $\mathrm{J} / \mathrm{cm}^{2}$ have recently been well described in the case of $\mathrm{Ne}$ atoms by a sequential multiphoton scheme [20].

In contrast to simultaneous multiphoton ionization, the number of pure sequential multiphoton processes per pulse does not explicitly depend on the pulse duration in cases where relaxation processes do not play a significant role [27]. Hence, spectra taken at the same radiant exposure should be identical. For the two spectra in Fig. 1, this is not the case. Thus, the multiphoton ionization of Xe at $93 \mathrm{eV}$ seems to have more than just simple sequential multiphoton processes. The reason might be the $4 d$ inner-shell giant resonance which dominates the photoionization of $\mathrm{Xe}$ at $93 \mathrm{eV}$ and leads to excited states with lifetimes of a few femtoseconds, decaying via Auger effect to higher charge states [36-38]. Similar mechanisms hold for the photoionization of $\mathrm{Xe}^{+}$to $\mathrm{Xe}^{6+}$ as the first steps of a sequential ionization scheme $[39,40]$. As a consequence, the $4 d$ vacancy is frequently refilled for further excitation. In the case of short photon pulses in the femtosecond regime, this sequence of inner-shell excitation and occupation via Auger relaxation may be interrupted ('frustrated') leading to a lower mean charge than at longer pulses. This idea has already been applied to explain the time-dependent multiphoton ionization of $\mathrm{Ne}$ in the harder X-ray regime, where the inner $1 s$ shell is addressed [16] and is confirmed by our ion yields in Fig. 2. For the higher charges $(\geq 4+)$, the values obtained at long pulses (a) are significantly higher and saturate at lower radiant exposure than at short pulses (b). The lower charges, on the other hand, may be reached without a second photon. Here, the ion yields at long and short pulses are much more similar.

The observed dependencies of the Xe ion yields on the pulse duration were further investigated by means of a bunch-resolved evaluation of our long-pulse data. While the comparison with the former results [22] in Fig. 1 and Fig. 2 was performed on a basis of averaging over FEL pulses of the same intensity but arbitrary bunch number, Fig. 3 shows the integrated charge state yields of $\mathrm{Xe}^{3+}$, $\mathrm{Xe}^{5+}, \mathrm{Xe}^{8+}$, and $\mathrm{Xe}^{10+}$ plotted against the radiant exposure and restricted to positions of the bunches at the beginning and at the end of a bunch train. At FLASH, the FEL pulse duration is affected by a not fully compensated beamloading effect due to a low-level radio frequency regulation. This leads to an amplitude and phase slope along the bunch train and, hence, to a corresponding change of the bunch compression, which results in increasing pulse duration from about $150 \mathrm{fs}$ to $250 \mathrm{fs}$ depending on the position of the 

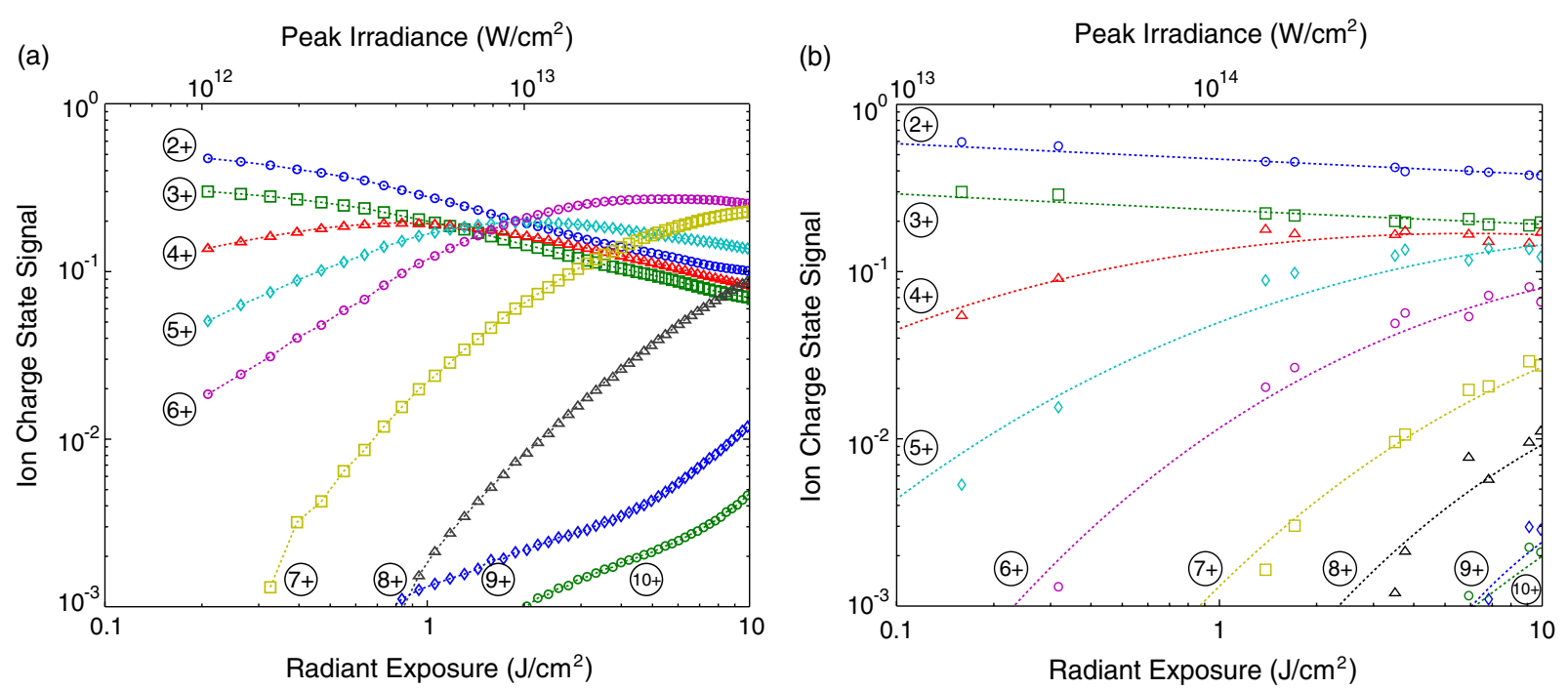

FIG. 2 (color online). Relative $\mathrm{Xe}^{2+}$ to $\mathrm{Xe}^{10+}$ ion yields (normalized to the sum of all charge state yields) as a function of radiant exposure: (a) current experiment and (b) data from [22].

bunch within a bunch train [41]. A former pump-probe study foreshadows this effect [42] but it is clearly reflected in Fig. 3 by the diverging ion yields of $\mathrm{Xe}^{5+}$ and $\mathrm{Xe}^{8+}$ for different bunch positions at high exposure.

The generation of $\mathrm{Xe}^{3+}$ states at $93 \mathrm{eV}$ photon energy is dominated by the resonant emission of an inner shell $4 d$ electron, starting from the Xe: $[\mathrm{Kr}] 4 d^{10} 5 s^{2} 5 p^{6}$ ground state, followed by two subsequent Auger decays [36-38]. It does not depend on the pulse duration (and bunch position) because just one photon is involved. With increasing exposure, the $\mathrm{Xe}^{3+}$ states are depleted due to subsequent ionization steps which cause the decrease of the $\mathrm{Xe}^{3+}$ signal in Fig. 3. Starting from $\mathrm{Xe}^{3+}$, resonant $4 d \rightarrow 4 f$ excitation is expected to be the main ionization channel [40] leading with some tens of femtoseconds lifetime via autoionization to $\mathrm{Xe}^{4+}$ and then, by absorption of a further photon, to $\mathrm{Xe}^{5+}$. The latter steps may be constrained in the case of shorter pulses approaching the lifetime of the preceding Auger and autoionization decays as previously discussed. This nicely explains the lower $\mathrm{Xe}^{5+}$ yield in Fig. 3 at shorter pulse duration of the early bunches compared to the longer pulses of the late bunches. The depletion of $\mathrm{Xe}^{5+}$ ions is less pronounced compared to $\mathrm{Xe}^{3+}$, as it is the relevance of subsequent ionization steps in the exposure range investigated. The yield of $\mathrm{Xe}^{8+}$ starts at almost zero below $1 \mathrm{~J} / \mathrm{cm}^{2}$ and increases highly nonlinearly with radiant exposure. The $\mathrm{Xe}^{8+}$ yields are higher for the shorter instead for the longer pulses. The most likely reason is that simultaneous multiphoton transitions are involved whose rates increase, according to Eq. (1), with shorter pulse duration $\tau$ at constant radiant exposure $H$. The transition energy from $\mathrm{Xe}^{7+}$ to $\mathrm{Xe}^{8+}$ amounts to more than $100 \mathrm{eV}$ [43] and, thus, exceeds our photon energy of $93 \mathrm{eV}$ so that at least two simultaneous photoabsorptions are required.
In Fig. 4, the time dependencies for the generation of $\mathrm{Xe}^{2+}$ to $\mathrm{Xe}^{9+}$ are summarized by three-dimensional contour plots of the yields, normalized to their mean values at the respective exposure and depicted with the bunch number on the horizontal axis and the radiant exposure on the vertical axis. For $\mathrm{Xe}^{2+}$ and $\mathrm{Xe}^{3+}$, the plots are almost flat, which means that the yields do not vary significantly with the bunch number or pulse duration at any radiant exposure and therefore indicates the underlying single photon processes. The yields generally increase with longer

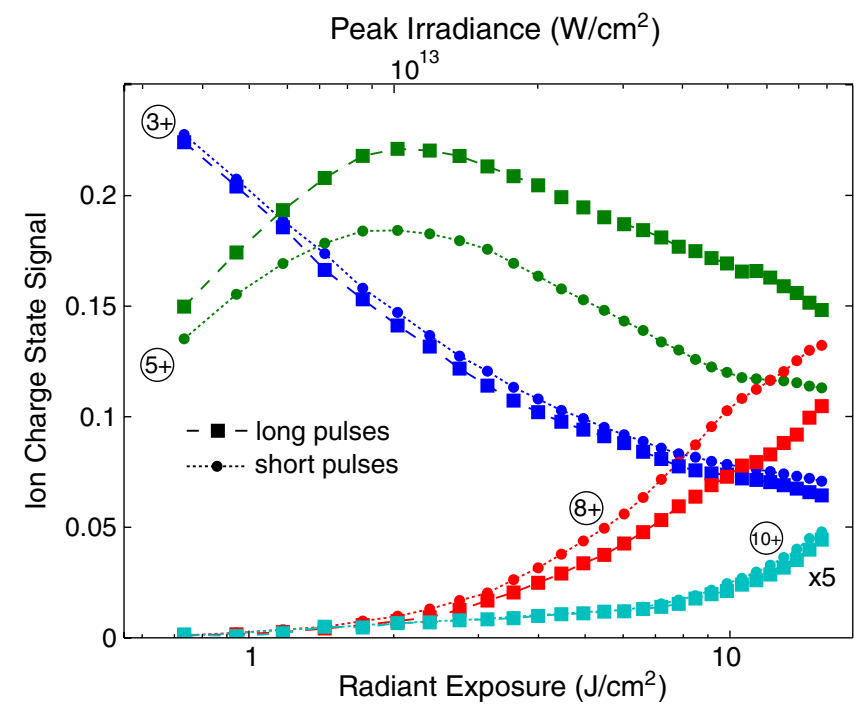

FIG. 3 (color online). Relative $\mathrm{Xe}^{3+}, \mathrm{Xe}^{5+}, \mathrm{Xe}^{8+}$, and $\mathrm{Xe}^{10+}$ ion yields (normalized to the sum of all charge state yields) as a function of the radiant exposure as observed from the early bunches (bunches 2 and 3, at slightly shorter FEL pulses) and from the late bunches (bunches 23 and 24, at slightly longer FEL pulses). 

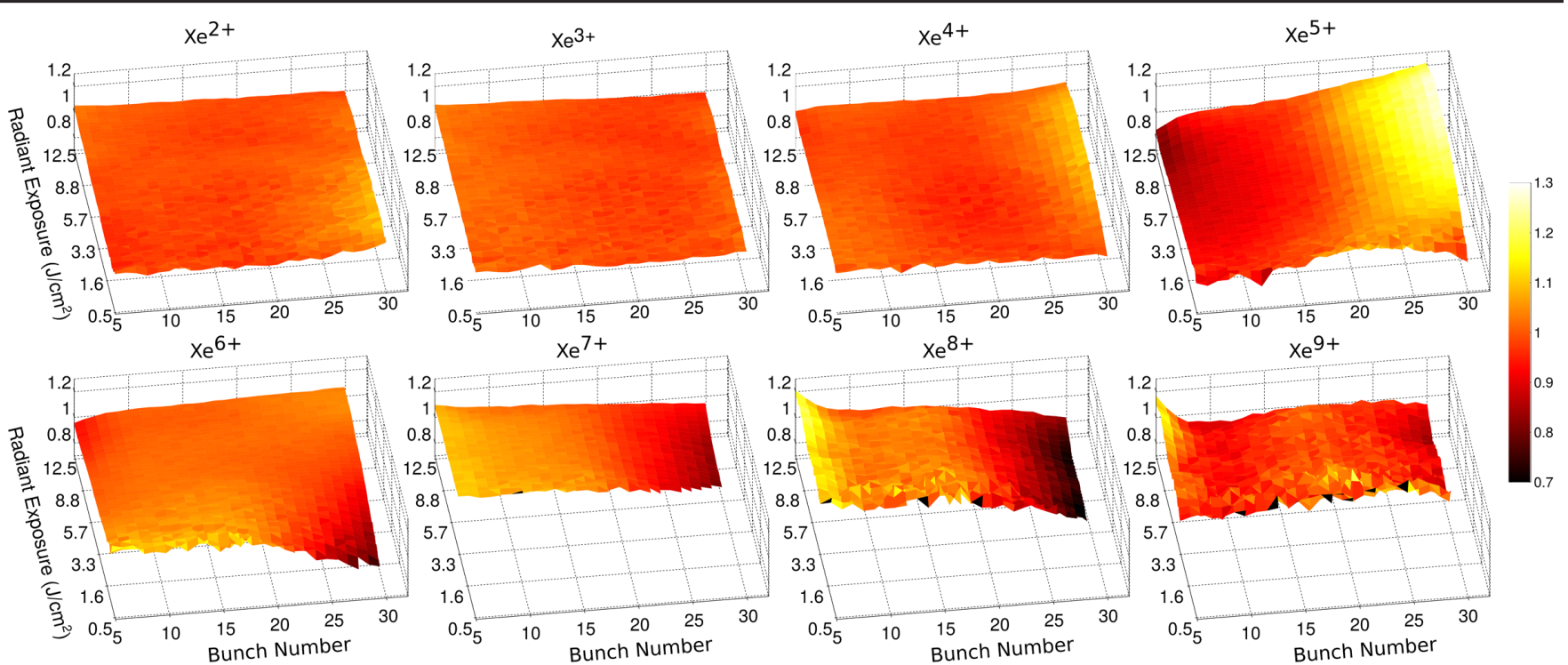

FIG. 4 (color online). Ionization yields for $\mathrm{Xe}^{2+}$ to $\mathrm{Xe}^{9+}$ as a function of radiant exposure and FEL bunch number, normalized to their mean values at the respective exposure level.

pulse duration at higher bunch number for $\mathrm{Xe}^{4+}$ and for $\mathrm{Xe}^{5+}$, which is explained by the gradual frustration, at shorter pulse lengths, of the previously discussed sequences of inner-shell excitation and occupation via Auger decay. The impact of simultaneous multiphoton processes connected with higher yields at shorter pulses according to Eq. (1) and lower yields at longer pulses and higher bunch numbers can already be observed at $\mathrm{Xe}^{6+}$ for the lower exposure values. This effect seems to be damped at higher exposure levels, probably due to saturation of the $\mathrm{Xe}^{6+}$ yield (see Fig. 2a). This observation is also valid for $\mathrm{Xe}^{7+}$. For $\mathrm{Xe}^{8+}$, the decrease of the yield at high bunch numbers is most distinct. Here, as for all charges greater than $6+$, the yields rise only at higher exposure levels [see Fig. 2(a)], so that their strongly varying low-exposure data are dominated by noise and omitted in Fig. 4 .

Of particular interest is the behavior of $\mathrm{Xe}^{9+}$ : its threedimensional contour plot in Fig. 4 is rather flat. Also for $\mathrm{Xe}^{10+}$, the yields in Fig. 3 obtained at the shorter and the longer pulses are equal within the statistical uncertainties. This might point to collective $4 d$ electron excitations for these higher charges because the strength of such a mechanism does not explicitly depend on the pulse duration. The scheme was already proposed for the particular multiphoton ionization of $\mathrm{Xe}[23,26]$ in the vicinity of the $4 d$ giant resonance [44-47] to explain the very high charge states in comparison to other rare gases and was subject of a substantial and controversial discussion [22-27]. Complementary to the perturbative approach of many individual photons interacting with many individual electrons within a complex sequential scheme, here the radiation field creates a sort of inner-atomic plasma oscillation and heating that leads to the emission of electrons. In the simplest approximation of a damped harmonic plasma oscillation, the energy transfer from a high-intensity EUV field to a single $\mathrm{Xe}$ atom is proportional to the radiant exposure $H$ without dependence on the pulse duration [26,27]. Naturally, this semiclassical approach applies only for higher intensities (and charges) on Xe in the vicinity of the $4 d$ giant resonance and is not in conflict with models based on perturbation theory for lower intensities and charges and other rare gases without a collective giant resonance. The higher rises of the $\mathrm{Xe}^{9+}$ and $\mathrm{Xe}^{10+}$ ion yields above $3 \mathrm{~J} / \mathrm{cm}^{2}$ in Fig. 2(a) also suggest the existence of two intensity regimes with obviously different mechanisms for the generation of the higher charges.

In conclusion, we were able to disentangle the main routes of multiphoton multiple ionization of $\mathrm{Xe}$ in the vicinity of the $4 d$ giant resonance up to a charge state of $\mathrm{Xe}^{10+}$ with our time-resolved iToF data. $\mathrm{Xe}^{2+}$ and $\mathrm{Xe}^{3+}$ were confirmed to be mainly generated by one-photon $4 d$ inner-shell ionization with subsequent Auger decay. $\mathrm{Xe}^{4+}$ and $\mathrm{Xe}^{5+}$ are due to further $4 d$ excitations followed by autoionization. The next steps of the ionization sequence seem to be concentrated in the outer shell of the respective ion with simultaneous multiphoton ionization playing a role for $\mathrm{Xe}^{6+}$ to $\mathrm{Xe}^{8+}$. Finally, the detected independence of $\mathrm{Xe}^{9+}$ and $\mathrm{Xe}^{10+}$ on the FEL pulse duration requires the collective $4 d$ electron excitations in the vicinity of the $4 d$ giant resonance of the $\mathrm{Xe}$ atom to explain the higher charges. This conclusion might be confirmed by future studies at different photon energies also outside of the $4 d$ giant resonance.

We thank the FLASH team for a very successful FEL operation. This work was supported by the Deutsche Forschungsgemeinschaft (DFG) under MA 2561/4-1, RI 804/5-1, TI 280/3-1, and SFB925/A3. 
*Present address: Paul Scherrer Institut, 5232 Villigen PSI, Switzerland.

[1] W. Ackermann et al., Nat. Photonics 1, 336 (2007).

[2] S. Di Mitri et al., Proc. IPAC 2011, 918 (2011).

[3] P. Emma et al., Nat. Photonics 4, 641 (2010).

[4] T. Shintake et al., Nat. Photonics 2, 555 (2008).

[5] D. Pile, Nat. Photonics 5, 456 (2011).

[6] C. Bostedt et al., Nucl. Instrum. Methods Phys. Res., Sect. A 601, 108 (2009).

[7] N. Berrah et al., J. Mod. Opt. 57, 1015 (2010).

[8] Intense X-ray Science-The first 5 years of FLASH, edited by H. Chapman, J. Ullrich, and J. M. Rost, J. Phys. B: At. Mol. Opt. Phys. B 43 (2010).

[9] Frontiers of Free-Electron Laser Science, edited by $\mathrm{P}$. Bucksbaum, T. Möller, and K. Ueda, J. Phys. B: At. Mol. Opt. Phys. B 46 (2013).

[10] M. Protopapas, C. H. Keitel, and P. L. Knight, Rep. Prog. Phys. 60, 389 (1997).

[11] N. Delone and V. Krainov, Multiphoton Processes in Atoms (Springer, New York, 2000).

[12] A. A. Sorokin, M. Wellhöfer, S. V. Bobashev, K. Tiedtke, and M. Richter, Phys. Rev. A 75, 051402(R) (2007).

[13] A. Rudenko et al., Phys. Rev. Lett. 101, 073003 (2008).

[14] M. Kurka et al., J. Phys. B: At. Mol. Opt. Phys. 42, 141002 (2009).

[15] K. Motomura et al., J. Phys. B: At. Mol. Opt. Phys. 42, 221003 (2009).

[16] L. Young et al., Nature (London) 466, 56 (2010).

[17] Y. Hikosaka et al., Phys. Rev. Lett. 105, 133001 (2010).

[18] B. Rudek et al., Nat. Photonics 6, 858 (2012).

[19] K. Motomura et al., J. Phys. B: At. Mol. Opt. Phys. 46, 164024 (2013).

[20] R. Guichard, M. Richter, J.-M. Rost, U. Saalmann, A. A. Sorokin, and K. Tiedtke, J. Phys. B: At. Mol. Opt. Phys. 46, 164025 (2013).

[21] H. Wabnitz, A. de Castro, P. Gürtler, T. Laarmann, W. Laasch, J. Schulz, and T. Möller, Phys. Rev. Lett. 94, 023001 (2005).

[22] A. A. Sorokin, S. Bobashev, T. Feigl, K. Tiedtke, H. Wabnitz, and M. Richter, Phys. Rev. Lett. 99, 213002 (2007).

[23] M. Richter, M. Amusia, S. Bobashev, T. Feigl, P. Juranić, M. Martins, A. Sorokin, and K. Tiedtke, Phys. Rev. Lett. 102, 163002 (2009).
[24] M. G. Makris, P. Lambropoulos, and A. Mihelič, Phys. Rev. Lett. 102, 033002 (2009).

[25] P. Lambropoulos, K. G. Papamihail, and P. Decleva J. Phys. B: At. Mol. Opt. Phys. 44, 175402 (2011).

[26] M. Richter, J. Phys. B: At. Mol. Opt. Phys. 44, 075601 (2011).

[27] M. Richter, A. A. Sorokin, and K. Tiedtke, Proc. SPIE Int. Soc. Opt. Eng. 8778, 877808 (2013).

[28] A. A. Sorokin et al., Appl. Phys. Lett. 89, 221114 (2006).

[29] K. Tiedtke et al., New J. Phys. 11, 023029 (2009).

[30] R. Mooshammer, private communication.

[31] R. Mitzner et al., Phys. Rev. A 80, 025402 (2009).

[32] Y. Inubushi et al., Phys. Rev. Lett. 109, 144801 (2012).

[33] C. Behrens, N. Gerasimova, Ch. Gerth, B. Schmidt, E. A. Schneidmiller, S. Serkez, S. Wesch, and M. V. Yurkov, Phys. Rev. ST Accel. Beams 15, 030707 (2012).

[34] K. Tiedtke et al., J. Appl. Phys. 103, 094511 (2008).

[35] P. Lambropoulos, Adv. At. Mol. Phys. 12, 87 (1976).

[36] U. Becker, T. Prescher, E. Schmidt, B. Sonntag, and H. E. Wetzel, Phys. Rev. A 33, 3891 (1986).

[37] T. Luhmann, Ch. Gerth, M. Groen, M. Martins, B. Obst, M. Richter, and P. Zimmermann, Phys. Rev. A 57, 282 (1998).

[38] M. Jurvansuu, A. Kivimäki, and S. Aksela, Phys. Rev. A 64, 012502 (2001).

[39] P. Andersen, T. Andersen, F. Folkmann, V. K. Ivanov, H. Kjeldsen, and J. B. West, J. Phys. B: At. Mol. Opt. Phys. 34, 2009 (2001).

[40] J. M. Bizau, C. Blancard, D. Cubaynes, F. Folkmann, J. P. Champeaux, J. L. Lemaire, and F. J. Wuilleumier, Phys. Rev. A 73, 022718 (2006).

[41] Martin Dohlus (private communication).

[42] U. Frühling et al., Nat. Photonics 3, 523 (2009).

[43] K. Yamakawa, Y. Akahane, Y. Fukuda, M. Aoyama, N. Inoue, H. Ueda, and T. Utsumi, Phys. Rev. Lett. 92, 123001 (2004).

[44] U. Fano and J. W. Cooper, Rev. Mod. Phys. 40, 441 (1968).

[45] F. A. Parpia, W. R. Johnson, and V. Radojević, Phys. Rev. A 29, 3173 (1984).

[46] M. Ya. Amusia, and J. P. Connerade, Rep. Prog. Phys. 63, 41 (2000).

[47] Giant Resonances in Atoms, Molecules, and Solids, edited by J.P. Connerade, J. M. Esteva, and R. C. Karnatak (Plenum Press, New York, 1987), and references therein. 